\title{
Optimal single 3T MR imaging sequence for HDR brachytherapy of cervical cancer
}

\author{
Claire Dempsey, PhD candidate',2, Jameen Arm, BSc (Med Rad Sci) ${ }^{3}$, Leah Best, BSc (Med Rad Sci) ${ }^{3}$, \\ Geetha Govindarajulu, MBBSl,4, Anne Capp, MBBS ${ }^{1,4}$, Peter O’Brien, MBBSl,4 \\ 'Department of Radiation Oncology, Calvary Mater Newcastle Hospital, ${ }^{2}$ School of Health Sciences, University of Newcastle, \\ ${ }^{3}$ Department of Radiology, Hunter New England Imaging, ${ }^{4}$ School of Medicine and Public Health, University of Newcastle, Australia
}

\begin{abstract}
Purpose: The superior image quality of 3 tesla (3T) magnetic resonance (MR) imaging in cervical cancer offers the potential to use a single image set for brachytherapy. This study aimed to determine a suitable single sequence for contouring tumour and organs at risk, applicator reconstruction, and treatment planning.

Material and methods: A 3T (Skyra, Siemens Healthcare AG, Germany) MR imaging system with an 18 channel body matrix coil generated HDR cervical cancer brachytherapy planning images on 20 cases using plastic-based treatment applicators. Seven different T2-weighted Turbo Spin Echo (TSE) sequences including both 3D and contiguous 2D scans based on sagittal, axial (transverse), and oblique planes were analysed. Each image set was assessed for total scanning time and usefulness in tumour localization via inter- and intra-observer analysis of high-risk clinical target volume (HR CTV) contouring. Applicator reconstruction in the treatment planning system was also considered.

Results: The intra-observer difference in HR CTV volumes between 2D and 3D axial-based image sets was low with an average difference of 3.1\% for each observer. 2D and 3D sagittal image sets had the highest intra- and inter observer differences (over 15\%). A 2D axial 'double oblique' sequence was found to produce the best intra- (average difference of $0.6 \%$ ) and inter-observer (mean SD of $9.2 \%$ ) consistency and greatest conformity (average 0.80 ).

Conclusions: There was little difference between 2D and 3D-based scanning sequences; however the increased scanning time of 3D sequences have potential to introduce greater patient motion artifacts. A contiguous $2 \mathrm{D}$ sequence based on an axial T2-weighted turbo-spin-echo (TSE) sequence orientated in all planes of the treatment applicator provided consistent tumour delineation whilst allowing applicator reconstruction and treatment planning.
\end{abstract}

Key words: cervix cancer, cervical cancer, magnetic resonance imaging, treatment planning, 3 Tesla.

\section{Purpose}

Three-dimensional (3D) image-based planning approaches to high dose rate (HDR) brachytherapy of cervical cancer have only been introduced in most departmental brachytherapy programs in the past decade, with magnetic resonance (MR) imaging now recommended as the imaging modality of choice. Current international recommendations state that T2-weighted, fast-spin-echo (FSE) or turbo-spin-echo (TSE) sequences to enable gross tumour to appear as high signal intensity masses should be taken in the para-axial, para-coronal, and para-sagittal planes when possible, for the ease of clinical target volume (CTV) visualisation [1-4]. Ideally, CTV contouring and applicator reconstruction should be performed on the same image sequence to reduce uncertainties in image fusion and patient/organ motion between different sequences. However, it has been acknowledged that there are often difficulties accurately visualising the applicator in T2-weighted MR images [3,4], particularly those with large slice thicknesses. In those instances, additional sequences such as a T1-weighted MR scan, a CT scan or even $\mathrm{X}$-ray images may be required $[3,4]$. These recommendations are based on studies conducted in brachytherapy treatment centres using low (0.1-0.5 tesla [T]) and high (1.0-1.5 T) magnetic resonance imagers, with acknowledgement that these studies contained no experience with higher (3T or higher) magnetic resonance imagers [3]. Several diagnostic imaging studies have shown that $3 \mathrm{~T} M R$ image of the female pelvis provides better image contrast and signal to noise ratio in the uterine cervix and vagina $[5,6]$, and there is continuing work in the evaluation of $3 \mathrm{~T}$ MR scans for brachytherapy imaging [7-9].

Brachytherapy, along with surgery and external beam radiotherapy (with or without chemotherapy) remains a significant part of the pattern of care for stage I-IVA cervical cancer [10]. HDR brachytherapy is generally performed post external beam irradiation, which provides greater initial tumour shrinkage, allowing the brachytherapy boost to 
sterilise any residual tumour while sparing bladder, rectum and small bowel from excessive dose.

Cervical cancer has a high-signal intensity on T2 weighted MR images [11,12]. Evaluation of T2 weighted MR images, taken at diagnosis, during treatment (external beam), after external beam (for brachytherapy), and up to 6 months follow up are often a good indication of how the tumour is responding to treatment. Unfortunately, any inflammation, fibrosis and oedema resulting from the external beam irradiation may also exhibit intermediate to high signal intensity on T2 weighted images [13,14]. GEC-ESTRO have recommended these areas of intermediate to high signal intensity be incorporated into either the high or intermediate risk clinical target volume (CTV) $[1,2,15]$. This makes MR image quality an important consideration in brachytherapy planning images and image resolution a major issue in accurate brachytherapy applicator and CTV determination.

In MR imaging, some important considerations are the signal-to-noise ratio (SNR) and image resolution. A 3T MR image has approximately double the SNR of a 1.5T MR image. By reducing slice thickness of the 3T MR scan, it is possible to maintain similar SNR to a 1.5T MR image, whilst increasing the resolution [16]. SNR is a central factor in HR CTV contouring and whilst resolution is critical in both HR CTV contouring and applicator reconstruction.

The purpose of this study was to determine a suitable 3T MR T2-weighted image sequence for 3D planning of HDR cervical cancer brachytherapy treatments. The increased image quality from a 3T MR image was hoped to produce a single image sequence for contouring tumour and organs at risk, applicator reconstruction, and treatment planning. A single image set will be more useful not only for treatment planning purposes but also a time-advantage to those departments that do not have immediate access to an MR scanner and may need to transfer a patient from theatre to radiology for treatment imaging.

\section{Material and methods \\ Patients}

The study was performed on 20 cases referred to the Radiation Oncology Department for the treatment of cervical cancer with brachytherapy. All patients had MR imaging performed for brachytherapy planning after plastic-based, MR compatible ring applicator (Ring CT/MR Applicator, Nucletron, an Elekta company) placement under ultrasound guidance and general anaesthesia. The age range of patients was between 31-88 years with a mean age of 54.3 years and all patients were rated FIGO (International Federation of Gynaecology and Obstetrics) stage IIB to IIIB. The range of contoured HR CTV for all patients was between 47.9 and $9.7 \mathrm{~cm}^{3}$ with a mean of $22.6 \mathrm{~cm}^{3}$. The range of HR CTV dimensions (L-R, S-I and A-P) were between $4.5,4.6$ and $5.5 \mathrm{~cm}$ and $1.9,1.7$, and $1.8 \mathrm{~cm}$, respectively.

All patients underwent external beam radiation therapy (EBRT) between 45 and $52.2 \mathrm{~Gy}$ in 1.8 or $2 \mathrm{~Gy}$ fractions. HDR brachytherapy (24 Gy in 8 Gy fractions prescribed to the $100 \%$ isodose line) was commenced either within the final week or within a week of finishing EBRT. Each insertion was given 1 week apart, with the applicator removed after each insertion and MR-based treatment planning occurring for each fraction.

All patients had cervical HDR brachytherapy applicators inserted under general anaesthetic, underwent MR scanning in radiology after leaving recovery and were then transported to the Department of Radiation Oncology for treatment.

\section{MR scanning technique}

A 3T (Skyra, Siemens Healthcare AG, Germany) MR imaging system with combination of an 18 channel body matrix and 32 channel phase array spine coil was used for generating HDR cervix brachytherapy planning images. Patients were positioned in supine head-first orientation with a body matrix coil placed over the pelvis. A small foam wedge was placed between the pelvis and coil to help reduce anterior abdominal wall motion. The pre-emptied bladder was filled with $100 \mathrm{ml}$ of saline for adequate distension of the bladder during imaging and reproducibility of bladder volume at treatment. An anti peristalsis agent was administered intravenously (Hyoscine butylbromide $20 \mathrm{mg}$ in $1 \mathrm{ml}$ ) to minimise ghosting artefacts from gross peristaltic movements during data acquisition. Scanning sequences that were part of the standard radiation therapy scanning practice in our department were considered for assessment. In collaboration with a radiologist and MR imaging specialist, a parallel imaging technique, generalized auto-calibrating partially parallel acquisition (GRAPPA) algorithm (Siemens Healthcare AG, Germany) with R factor of 2 was incorporated in all sequences to reduce scan times to tolerable limits. Spatially selective saturation bands were used superiorly and anteriorly on all sequences to suppress the signal from inflowing spins and reduce motion related artefacts. T2 weighted turbo spin echo (TSE) sequences were selected without the application of IV contrast media in keeping with international recommendations [3]. Both 3D T2-weighted sampling perfection with application-optimised contrast and different flip-angle evolutions (SPACE, Siemens Healthcare AG, Germany) (3D T2) sequences and contiguous 2D T2-weighted TSE sequences (2D T2) scans were performed for comparison in sagittal, axial, and oblique planes tilted to correspond to the treatment applicator. A total of seven sequences with various orientations were analysed.

Firstly, a 3-plane half-Fourier acquisition single-shot turbo spin-echo (HASTE, Siemens Healthcare AG, Germany) localizer was taken. This HASTE scan was used for planning the axial and oblique-axial sequences oriented to correspond to the treatment applicator.

Initially, a 2D T2 weighted TSE (2D T2) sequence was obtained in the sagittal plane between the obturator muscles, and a 2D T2 sequence was positioned and acquired in a true axial plane and reviewed for both applicator and tissue delineation. A 3D T2 volumetric sequence was also performed in a true axial plane with an isotropic 3D T2 volumetric sequence acquired in the sagittal plane.

Both a 2D T2 sequence and a 3D T2 volumetric sequence were planned and acquired para-axially with the scan- 


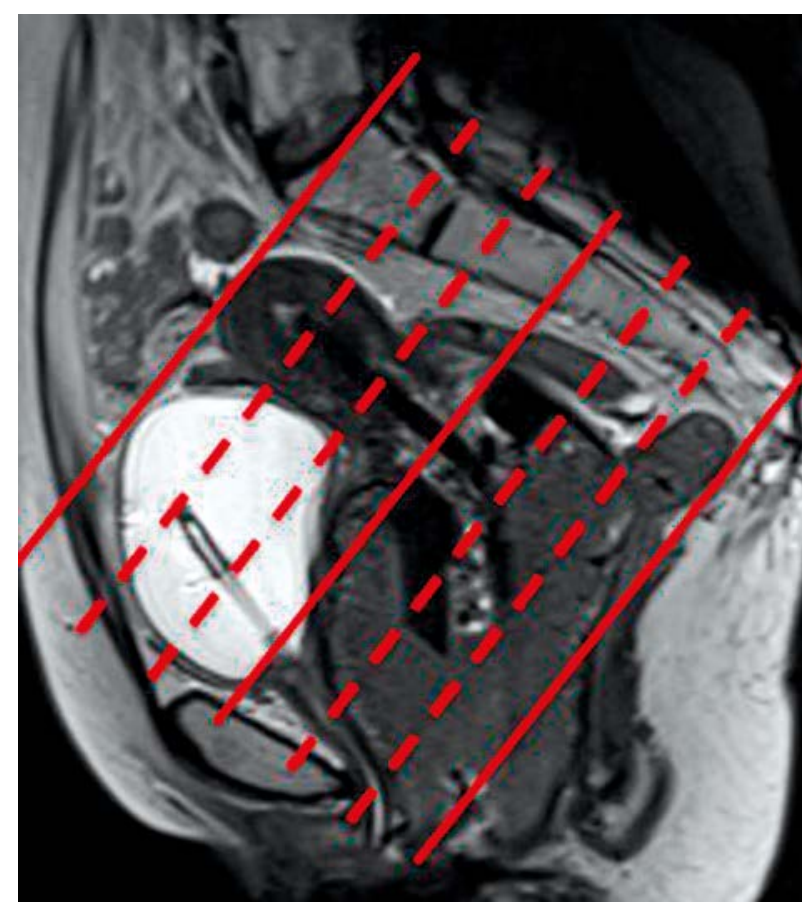

Fig. 1. Axial-oblique plane, aligned parallel to the plane of the ring in the sagittal scan

ning plane aligned parallel to the ring surface from the sagittal HASTE scan (axial oblique, see Fig. 1). Another 2D $\mathrm{T} 2$ sequence was then obtained with the scanning plane parallel to the ring surface, based on both the sagittal and the coronal reconstructions of the HASTE localiser (axial double oblique, see Fig. 2).

All scan volumes included the uterine fundus superiorly and the distal end on the treatment applicator inferiorly. All scanning sequences were designed to have slice thicknesses less than $2 \mathrm{~mm}$ to aid in 3D reconstruction in the brachytherapy treatment planning system. Detailed scanning parameters are listed in Table 1.

\section{Image analysis}

After importation into the treatment planning system (Oncentra Brachy version 4.2 and 4.3, Nucletron, an Elekta Company, Sweden), the images were initially evaluated by an experienced Medical Physicist with regards to the ability to reconstruct the treatment applicators. An applicator library (Applicator Modelling module in Oncentra version 4.2 and 4.3, Nucletron, an Elekta Company, Sweden) was used in the reconstruction of each applicator. In this module, a minimum of 3 pre-defined points (anchor points) are required in order to reconstruct the applicator. An applicator reconstruction was deemed 'successful' if, after placement of the anchor points and any additional manual manipulation, the reconstructed applicator was clearly seen to lie within $\pm 2 \mathrm{~mm}$ of the visualised applicator in the MR images. This value was chosen as it represents the tolerance placed on applicator reconstruction defined by the AAPM task group 56 [17].

Each image set was then assessed for total scanning time and usefulness in tumour localization via inter- and

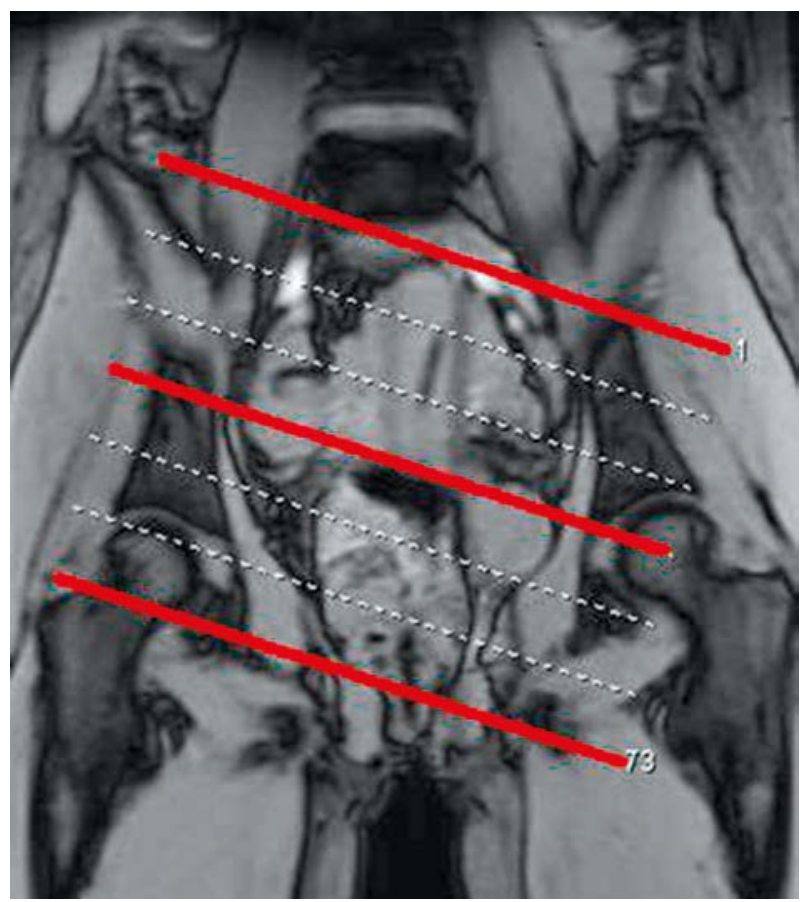

Fig. 2. Axial double-oblique additional plane, aligned parallel to the plane of the ring in the coronal localizer scan

intra-observer analysis of high-risk clinical target volume (HR CTV) contouring. HR CTV contours were generated based on GEC-ESTRO recommendations [1,2] for each data set by three radiation oncologists experienced in gynaecological brachytherapy, who were blinded to the scanning technique. Anatomical changes and geographical shifts between imaging sequences were also examined, along with visual differences between the contouring and planning modules of the brachytherapy treatment planning system.

Intra-observer analysis (comparing contours from a single radiation oncologist between different imaging sequences) was performed by determining the relative volume difference from the mean in HR CTVs between image sets for the same patient. These values were then averaged across all patients in the study.

Inter-observer analysis (comparing contours from all three radiation oncologists for a single image sequence) was performed not only by determining the relative volume difference in HR CTVs, but also by applying a conformity index (CI) based on common and encompassing volume for all 3 observers. The CI was determined based on the work by Kouwenhoven et al. that allowed a CI to be determined independent of the number of observers [18]. The CI is given by the following equation (1):

$$
\mathrm{CI}=\frac{\sum_{k=1}^{K} k(k-1) V_{k}}{2(K-1) \sum_{k=1}^{K} k V_{k}-\sum_{k=1}^{K} k(k-1) V_{k}}
$$

where: $K$ - total number of observers, $V_{k}$ - volume encompassed by $k$ observers. 


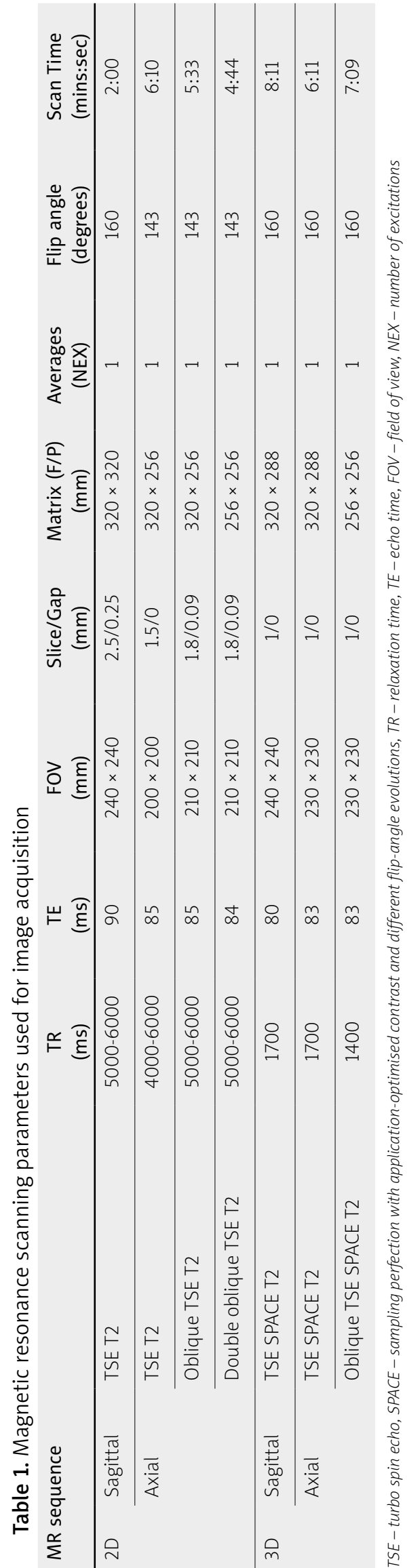

\section{Results}

All sequences were able to be imported into the brachytherapy treatment planning system, however, those sequences that contained oblique planes required any automatic image fixing to be disabled. Due to the use of plastic-only treatment applicators, image distortion effects that are known to be present particularly in 3T MR imaging [7] were negligible. Visualization and eventual reconstruction of the applicator by the medical physicist resulted in agreement between the applicator model and the MR images of $\pm 4 \mathrm{~mm}$ at first pass, and less than $\pm 2 \mathrm{~mm}$ after manual manipulation for all imaging sequences.

Table 1 indicates the scanning times for each of the sequences tested. A double oblique axial T2 3D scan was not used in this study as the scanning time was considered excessive at close to 10 minutes. The double oblique axial T2 2D scan had the fastest scan time after the T2 2D sagittal scan, however the sagittal scan had a much larger slice thickness ( $2.5 \mathrm{~mm}$ compared with $1.8 \mathrm{~mm}$ ).

Results for intra- and inter-observer HR CTV contouring are shown in Table 2. For intra-observer HR CTV analysis, both 2D and 3D sagittal image sequences had the worst consistency across all radiation oncologists with an average difference of $19.8 \%$ and $15.1 \%$, respectively. The best volume consistency across all radiation oncologists was for 2D axial double oblique and 3D axial oblique image sequences, with an average difference of less than $1 \%$, and there was an average difference of $3.1 \%$ across all 2D and 3D axial image sequences.

When comparing volume sizes only for inter-observer HR CTV analysis, the mean relative SD across all cases and sequences was $24.2 \%$. The $2 \mathrm{D}$ axial double oblique image sequence had the best consistency with a mean relative SD of $9.2 \%$ and the $2 \mathrm{D}$ sagittal the worst with a mean relative SD of $37 \%$. The $2 \mathrm{D}$ axial double oblique image sequence had the highest conformity index (mean 0.80 , range $0.72-0.91$ ), while the $2 \mathrm{D}$ axial image had the worst (mean 0.55 , range $0.54-0.75$ ).

\section{Discussion}

Applicator reconstruction in MR images is filled with inherent uncertainty. Problems may arise with reconstruction from MR imaging scans as it is very difficult to visualize the internal structure of the applicator to confirm the source path [4]. Whilst markers and applicator libraries can be effective in detailing the source path, there are uncertainties in reconstruction that relate directly to the slice thickness of the imaging scans used. Reconstruction uncertainties have been noted to be greater in the direction perpendicular to slice orientation which is commonly linked to the large pixel size or slice thickness in this direction $[15,19]$. It is recommended that slice thickness be as small as possible without increasing MR image acquisition time too greatly as this increases the risk of patient motion during the scan [19]. Reducing the MR slice thickness to less than $2 \mathrm{~mm}$ can help minimize random error in applicator reconstruction. This type of scan is only achievable in high strength (3T) MR scanners, as the loss of SNR, increased scanning time, and increased 
Table 2. Intra- and inter-observer differences for different 3T T2 MR scanning sequences A. Intra-observer difference

\begin{tabular}{|c|c|c|c|c|c|c|}
\hline \multicolumn{3}{|c|}{ MR sequence type } & \multicolumn{4}{|c|}{ Mean \% difference } \\
\hline & & & \multirow{2}{*}{$\begin{array}{c}\text { Observer } 1 \\
35.7\end{array}$} & \multirow{2}{*}{$\begin{array}{c}\text { Observer } 2 \\
9.3\end{array}$} & \multirow{2}{*}{$\begin{array}{c}\text { Observer } 3 \\
14.5\end{array}$} & \multirow{2}{*}{$\begin{array}{c}\begin{array}{c}\text { Average over } \\
\text { 3 observers }\end{array} \\
19.8\end{array}$} \\
\hline $2 \mathrm{D}$ & Sagittal & TSE T2 & & & & \\
\hline & Axial & TSE T2 & 6.4 & 0.3 & 11.7 & 6.1 \\
\hline & & Oblique TSE T2 & 5.4 & 1.2 & 6.1 & 4.2 \\
\hline & & Double oblique TSE T2 & 1.2 & 0.3 & 0.3 & 0.6 \\
\hline \multirow[t]{3}{*}{$3 \mathrm{D}$} & Sagittal & TSE SPACE T2 & 22.8 & 9.5 & 12.9 & 15.1 \\
\hline & Axial & TSE SPACE T2 & 1.0 & 1.7 & 0.1 & 0.9 \\
\hline & & Oblique TSE SPACE T2 & 6.5 & 1.0 & 4.1 & 3.9 \\
\hline \multicolumn{7}{|c|}{ B. Inter-observer differences } \\
\hline \multicolumn{3}{|c|}{ MR sequence type } & \multicolumn{2}{|c|}{$\%$ SD of HR CTV volume } & \multicolumn{2}{|c|}{ Conformity index } \\
\hline & & & Mean & Range & Mean & Range \\
\hline \multirow[t]{4}{*}{ 2D } & Sagittal & TSE T2 & 37.0 & $28.7-48.9$ & 0.59 & $0.40-0.68$ \\
\hline & Axial & TSE T2 & 32.5 & $17.7-47.3$ & 0.55 & $0.45-0.75$ \\
\hline & & Oblique TSE T2 & 30.6 & $14.1-68.3$ & 0.63 & $0.58-0.74$ \\
\hline & & Double oblique TSE T2 & 9.2 & $2.9-12.4$ & 0.80 & $0.72-0.91$ \\
\hline \multirow[t]{3}{*}{$3 D$} & Sagittal & TSE SPACE T2 & 19.8 & $9.8-31.3$ & 0.71 & $0.60-0.79$ \\
\hline & Axial & TSE SPACE T2 & 28.1 & $16.6-46.8$ & 0.60 & $0.61-0.72$ \\
\hline & & Oblique TSE SPACE T2 & 12.3 & $4.1-13.5$ & 0.74 & $0.70-0.78$ \\
\hline
\end{tabular}

potential for patient motion $[15,19]$ would be too great for lower strength MR scanners.

The GEC ESTRO recommendations for 3D image guided brachytherapy of the cervical cancer are acknowledged as being a well-defined method for creating target and organ at risk volumes with which to optimize a patient treatment [1-3]. Even with these guidelines, there are still uncertainties and different interpretations of clinical and pathological disease, implying that it is not possible to have complete agreement between observers.

There have been several studies into inter-observer differences for target volume delineation in cervical cancer brachytherapy. Petrič et al. (2013) performed a multi-institutional study with 10 observers and 6 cases. The interobserver relative SD for the HR CTV was up to $42 \%$ with an average conformity index of 0.74 [20]. In 2008, Petrič et al. investigated HR CTV contour differences between axial and para-axial (oblique axial) image sets for a $0.2 \mathrm{~T}$ MR scanner. The inter-observer conformity index for the axial and para-axial sequences was given as 0.79 and 0.78 , respectively [21]. Similar results were seen in another study in 2009 [22]. Our study highlights the effect that even a single 'tilt' on a scanning plane can do for contouring reliability. There was a large difference between the 2D T2 axial oblique and 2D T2 axial double oblique, $30.6 \%$ mean relative SD dropping to $9.2 \%$ mean relative $\mathrm{SD}$. This indicates that all scanning planes must be care- fully and individually designed before a scan takes place and highlights the importance of testing multiple imaging planes, when setting up a MR imaging program for cervical cancer brachytherapy.

The dosimetric impact of inter-observer variability has been studied, with levels of uncertainty in HR CTV contouring causing variation of $\pm 5 \mathrm{~Gy}$ in $\mathrm{D}_{90}$ over the total course of radiotherapy (external beam and brachytherapy) or an uncertainty of $9 \%$ for a single intracavitary brachytherapy fraction [23]. This study used images from MR scanners with magnet strength $0.2 \mathrm{~T}$ and $1.5 \mathrm{~T}$. The mean relative SD of the contoured HR CTV was 19\% (across 10 observers and 6 cases). Interestingly, the patients scanned on the $0.2 \mathrm{~T}$ MR scanner had lower mean SD than the patients scanned on the $1.5 \mathrm{~T}$ (17\% vs. $21 \%)$. This is compared to $9.2 \%$ for $3 \mathrm{~T}$ MR $2 \mathrm{D}$ axial double oblique scans in the present study.

In any case, it is ideal to eliminate as much as possible any potential causes of observer contouring variation. There have been several studies that have looked into ways to reduce this variation $[24,25]$. In these they recommended multiple imaging modalities, increasing image quality, extensive training in target contouring, and following written protocols. The latter two recommendations can be addressed using the GEC-ESTRO contouring criteria [1-3], while the second recommendation can be achieved by optimizing the choice of scanning sequence 
and the quality of the imaging system. Petrič et al. (2012) studied the differences in contours between 2D multi-planar scanning sequences (as recommended by GEC ESTRO [3]) and a 3D single scanning sequence for a 1.5T MR scanner and found little difference in target contours for 2 observers and 14 patients [26]. The results of our study also indicate that there is minimal difference in HR CTV contours when completed on 2D or 3D image sets using a 3T MR scanner. Several diagnostic studies have also indicated little difference in image quality between 2D T2 weighted and 3D SPACE T2 weighted images. The main benefit of using a 3D SPACE T2 image set was defined as the time saving over using multi-planar 2D T2 image sequences [27-29]. If the 2D multi-planar image set could be reduced to a single image set without compromising image quality in 3-dimensional reconstructions, the reduction in scanning time could be even further improved over the 3D T2 sequence.

This study has examined 7 different 3T MR scanning sequences in order to determine which single image set provides the best consistency for target delineation, both intra- and inter-observer. Whilst a limitation to this study is the size of the number of observers $(N=3)$, this work still highlights the variability that the choice of scanning sequence can produce. The organ at risk (OAR) contour variation was not performed in this study. Further work investing the possible changes to OAR volume definition for varying $3 T$ MR sequences will be carried out.

Patient shifts sometimes seen between scanning sequences can vary the position of the applicator relative to the CTV and OAR, resulting in inconsistencies if using multiple scanning sequences to contour and plan. This would be a particular concern if one sequence was used for contouring and another sequence used for planning as the relationship between source position and CTV or OAR may have changed between scanning sequences. Sagittal and coronal images as recommended by GEC ESTRO [1-3] are currently mostly used for verification purposes as treatment planning software does not allow users to contour a single structure across multiple imaging planes. A standard sagittal 2D T2 TSE sequence scan can also be performed if time permits, and there appears to be little patient motion during the para-axial scan. This scan can then be a useful tool in single plane verification of the CTV and OAR contours if required.

Using the 2D axial double oblique scanning sequence, the total scanning time for each patient can be reduced to less than 7 minutes. Even with a bladder filling protocol and administration of anti-peristalsis agent in the scanning room, patients can be in and out of the MR scanning room in less than 15 minutes. This has multiple benefits to the patient with less time isolated inside the scanning room and less time required to be lying compliant on the scanning table. This is particularly important in smaller centres that do not have the capability to generate treatment planning images, whilst the patient is under a general anaesthetic. The rapid turnaround of patients also allows a more efficient use of staff time and equipment resources.

\section{Conclusions}

Different MR scanning sequences can greatly affect the disparity in HR CTV contours between Radiation Oncologists. Intra and inter-observer variation in HR CTV contouring suggests a 2D T2 TSE axial sequence oriented in all planes to the treatment applicator (double oblique) on a 3T MR scanner, allows more accurate and uncomplicated tumour determination in brachytherapy treatment planning of cancer of the cervix. Applicator visualization is sufficient for planning requirements and the thinner slice thickness reduces the expected uncertainty in applicator reconstruction. The ability to contour, plan, and optimize the brachytherapy treatment using a single image set that is orientated in the most useful plane for these tasks is a great benefit. This imaging sequence was tested on 20 cases, but has since been successfully applied to another 35 cases, including patients using an interstitial/ intracavitary applicator (interstitial ring CT-MR applicator set, Nucletron, an Elekta Company, Sweden).

Minimizing scanning time through elimination of excess scanning sequences implies greater patient throughput via reduced demand on the MR scanner and lessens patient discomfort via reducing the time required to be on the MR scanning table. This is a critical factor in departments that do not have access to an MR unit whilst the patient is under general anesthetic.

\section{Acknowledgements}

This work is based on an abstract that was presented at the Brachytherapy World Congress (Barcelona, May 2012).

\section{Disclosure}

Authors report no conflict of interest.

\section{References}

1. Haie-Meder C, Pötter R, Van Limbergen E et al. Recommendations from Gynaecological (GYN) GEC ESTRO Working Group (I): concepts and terms in 3D image-based 3D treatment planning in cervix cancer brachytherapy with emphasis on MRI assessment of GTV and CTV. Radiother Oncol 2005; 74: 235-245.

2. Pötter R, Heie-Meder C, van Limbergen E et al. Recommendations from the Gynaecological (GYN) GEC ESTRO Working Group (II): concepts and terms in 3D image-based treatment planning in cervix cancer brachytherapy - 3D volume parameters and aspects of 3D image-based anatomy, radiation physics, radiobiology. Radiother Oncol 2006; 78: 67-77.

3. Dimopoulos J, Petrow P, Tanderup K et al. Recommendations from Gynaecological (GYN) GEC-ESTRO Working Group (IV): Basic principles and parameters for MR imaging within the frame of image based adaptive cervix cancer brachytherapy. Radiother Oncol 2012; 103: 113-122.

4. Hellebust TP, Kirisits C, Berger D et al. Recommendations from Gynaecological (GYN) GEC-ESTRO Working Group: Considerations and pitfalls in commissioning and applicator reconstruction in 3D image-based treatment planning of cervix cancer brachytherapy. Radiother Oncol 2010; 96: 153-160.

5. Kataoka M, Kido A, Koyama T et al. MRI of the female pelvis at $3 \mathrm{~T}$ compared to 1.5T: Evaluation on high-resolution T2-weighted and HASTE images. J Magn Reson Imaging 2007; 25: 527-534. 
6. Hussain SM, van den Bos IC, Oliveto JM et al. MR imaging of the female pelvis at 3T. Magn Reson Imaging Clin N Am 2007; 14: 537-544.

7. Kim Y, Muruganandham M, Modrick JM et al. Evaluation of artifacts and distortions of titanium applicators on 3.0-Tesla MRI: feasibility of titanium applicators in MRI-guided brachytherapy for gynecological cancer. Int J Radiat Oncol Biol Phys 2011; 80: 947-955.

8. Kapur T, Egger J, Damato A et al. 3-T MR-guided brachytherapy for gynecologic malignancies. Magn Reson Imag 2012; 30: 1279-1290.

9. Anderson JW, Xia J, Flynn RT et al. High resolution 3T MRIguided conformal brachytherapy for cervical cancer: consequences of different high-risk CTV sizes. J Contemp Brachytherapy 2013; 5: 101-109.

10. Viswanathan AN, Beriwal S, De Los Santos JF et al. American Brachytherapy Society consensus guidelines for locally advanced carcinoma of the cervix. Part II: High-dose-rate brachytherapy. Brachytherapy 2012; 11: 47-52.

11. Hricak H, Lacey CG, Sandles LG et al. Invasive cervical carcinoma: Comparison of MR imaging and surgical findings. Radiology 1988; 166: 623-631.

12. Togashi K, Nishimura K, Sagoh T et al. Carcinoma of the cervix: staging with MR imaging. Radiology 1989; 171: 245-251.

13. Ebner F, Kressel HY, Mintz MC et al. Tumour recurrence versus fibrosis in the female pelvis Differentiation with MR imaging at 1.5 T. Radiology 1988; 166: 333-340.

14. Hatano K, Sekiya Y, Araki H et al. Evaluation of the therapeutic effect of radiotherapy on cervical cancer using magnetic resonance imaging. Int J Radiat Oncol Biol Phys 1999; 45: 639-644.

15. Dimopoulos J, Schard G, Berger D et al. Systematic evaluation of MRI findings in different stages of treatment of cervical cancer: potential or MRI on delineation of target, pathoanatomic structures and organs at risk. Int J Radiat Oncol Biol Phys 2006; 64: 1380-1388.

16. Willinek WA, Schild HH. Clinical advantages of 3.0T MRI over 1.5T. Euro J Radiol 2008; 65: 2-14.

17. Nath R, Anderson LL, Meli JR et al. Code of practice for brachytherapy physics: report of the AAPM Radiation Therapy Committee Task Group No. 56. American Association of Physicists in Medicine. Med Phys 1997; 24: 1557-1598.

18. Kouwenhoven E, Giezen M, Struikmans H. Measuring the similarity of target volume delineations independent of the number of observers. Phys Med Biol 2009; 54: 2863-2873.

19. Tanderup K, Hellebust TP, Lang S et al. Consequences of random and systematic reconstruction uncertainties in 3D image based brachytherapy in cervical cancer. Radiother Oncol 2008; 89: 156-163.

20. Petrič P, Hudej R, Rogelj P et al. Uncertainties of target volume delineation in MRI guided adaptive brachytherapy of cervix cancer: A multi-institutional study. Radiother Oncol 2013; 107: 6-12.

21. Petrič P, Dimopoulos J, Kirisits C et al. Inter- and intraobserver variation in HR-CTV contouring: intercomparison of transverse and paratransverse image orientation in 3D-MRI assisted cervix cancer brachytherapy. Radiother Oncol 2008; 89: 164-171.

22. Dimopoulos JCA, De Vos V, Berger D et al. Inter-observer comparison of target delineation for MRI-assisted cervical cancer brachytherapy: application of the GYN GEC-ESTRO recommendations. Radiother Oncol 2009; 91: 166-172.

23. Hellebust TP, Tanderip K, Lervåg C et al. Dosimetric impact of interobserver variability in MRI-based delineation for cervical cancer brachytherapy. Radiother Oncol 2013; 107: 13-19.

24. Njeh CF. Tumor delineation: the weakest link in the search for accuracy in radiotherapy. J Med Phys 2008; 33: 136-140.
25. Weiss E, Richter S, Krauss $\mathrm{T}$ et al. Conformal radiotherapy planning of cervix carcinoma: differences in the delineation of the clinical target volume: A comparison between gynaecologic and radiation oncologists Radiother Oncol 2003; 67: 87-95.

26. Petrič P, Hudej R, Rogelj P et al. Comparison of 3D MRI with high sampling efficiency and 2D multiplanar MRI for contouring in cervix cancer brachytherapy. Radiol Oncol 2012; 46: 242-251.

27. Rosenkrantz AB, Neil J, Kong X et al. Prostate cancer: Comparison of 3D T2-weighted with conventional 2D T2-weighted imaging for image quality and tumor detection. Am J Roentgenol 2010; 194: 446-452.

28. Proscia N, Jaffe TA, Neville AM et al. MRI of the pelvis in women: $3 \mathrm{D}$ versus 2D T2-weighted technique. Am J Roentgenol 2010; 195: 254-259.

29. Hecht EM, Yitta S, Lim RP et al. Preliminary clinical experience at $3 \mathrm{~T}$ with a $3 \mathrm{D}$ T2-weighted sequence compared with mulitplanar 2D for evaluation of the female pelvis. Am J Roentgenol 2011; 197: 346-352. 